\title{
Tuberculosis in the 21st century
}

\author{
Jon S Friedland
}

Tuberculosis (TB) is one of the most fascinating infectious diseases of the 21st century. The pathogen infects a third of the world's population and manifestations of disease are protean. There are difficulties in prevention, diagnosis and treatment of TB. This article will focus on areas that I consider to be of particular current interest.

\section{TB epidemiology}

In 1993, the World Health Organization (WHO) declared TB a global emergency but disappointingly little has changed other than the emergence of increasingly drug-resistant organisms. WHO figures from 2009 (www.who.int/tb/country/en/) show that the highest incidence of TB is still in sub-Saharan Africa where infection rates of over 300/100,000 are common often in association with underlying HIV infection. However, if absolute numbers are considered, it is clear that the burden of TB is in the densely populated regions of the world such as China and India (Fig 1).

At any one time, approximately nine million people worldwide have active disease and approaching two million die each year. As a disease burden, these numbers are large. However, in the context of the 1.7 billion infected with Mycobacterium tuberculosis (MTb) less than $1 \%$ of those people have active disease and a tiny fraction die. Thus, in normal circumstances the human immune system copes very well with controlling TB while allowing spread of disease.

Closer to home in UK, the Health Protection Agency reported over 9,000 new cases of TB in 2009, a 5.5\% increase on the previous year. The UK incidence of TB is 14.9 cases per 100,000 population. However, this disguises huge variation. TB is concentrated in urban areas and $41 \%$ disease is in London. Seventythree per cent of TB cases in the UK are from patients who were born overseas. In the UK-born population, although much TB occurs in communities associated with non-UK-born individuals, the majority of patients are white Caucasians even though the rate per 100,000 population is very low at around three. One fact which is not widely appreciated is that $43 \%$ of UK TB is extrapulmonary affecting brain, lymph nodes, bones, the gastrointestinal system and other organs. TB in the UK has a significant mortality; there were around 500 deaths in the late 1980s, a figure now heading down below 350 .

This article is based on a lecture given by Jon S Friedland, head of infectious diseases and immunity, Imperial College London, at the North West Region Royal College of Physicians Update in Medicine on 15 April 2010

\section{Difficulties in TB diagnosis}

Diagnosis of TB is frequently fraught with difficulty. In affluent countries, the disease is often not considered and symptomatic patients may attend primary care on several occasions receiving standard antibiotics before investigations are instigated. In poorer parts of the world, failure to diagnose $\mathrm{TB}$ is a major problem resulting in a considerable number of case under ascertainment. Diagnosis is often based on microscopy (as initially recommended for all international directly observed therapy shortcourse programmes) which has about $50 \%$ sensitivity of culture. As a result, there are few data on drug susceptibility. Unrecognised drug resistance leads to prolonged infectivity, spread of infection and adverse clinical outcomes including death. In large parts of the world, there is no infrastructure for TB diagnosis. There is a need for a test that is simple, robust, rapid and provides details of drug resistance to at least isoniazid and rifampicin, the two main anti-mycobacterial drugs.

Times are starting to change and simple but powerful molecular diagnostic tests are emerging; ${ }^{1}$ these are reviewed in more detail elsewhere. ${ }^{2}$ Issues around these tests, such as cost and care of equipment, remain in many parts of the world. Transport of samples from rural areas through several layers of the system to a centralised laboratory is in itself a major challenge.

One answer to the need for a cheap and rapid test for TB and drug sensitivity is the microscopic observation drug susceptibility assay or MODS. ${ }^{3,4}$ The principle is simple and depends on the culture of decontaminated sputum samples in broth and the examination of the broth under an inverted light microscope to detect the characteristic pattern of growth of MTb (Fig 2). The critical part of the test is the incorporation of anti-TB drugs at the outset to provide direct susceptibility testing of infected samples. In contrast, most other tests rely on first detection of the organism and then secondary or indirect drug susceptibility testing which inevitably delays diagnosis of drug resistance. MODS is more sensitive than not only traditional techniques such as Lowenstein-Jenson (LJ) cultures, but also modern MB bactec machines. The median time to diagnosis with MODS was seven days, with MB bactec was 13 days and with LJ slopes about 24 days. ${ }^{5}$ However, the key point is that with MODS at the time of diagnosis drug susceptibilities are available whereas with the other two tests a further round of investigation was required before drug susceptibilities can be obtained. Most patients are diagnosed by MODS within two weeks. MODS is similarly efficient irrespective of the burden of $\mathrm{TB}$ whereas MB bactec was much faster in patients who were smear sputum positive compared to those who were sputum 


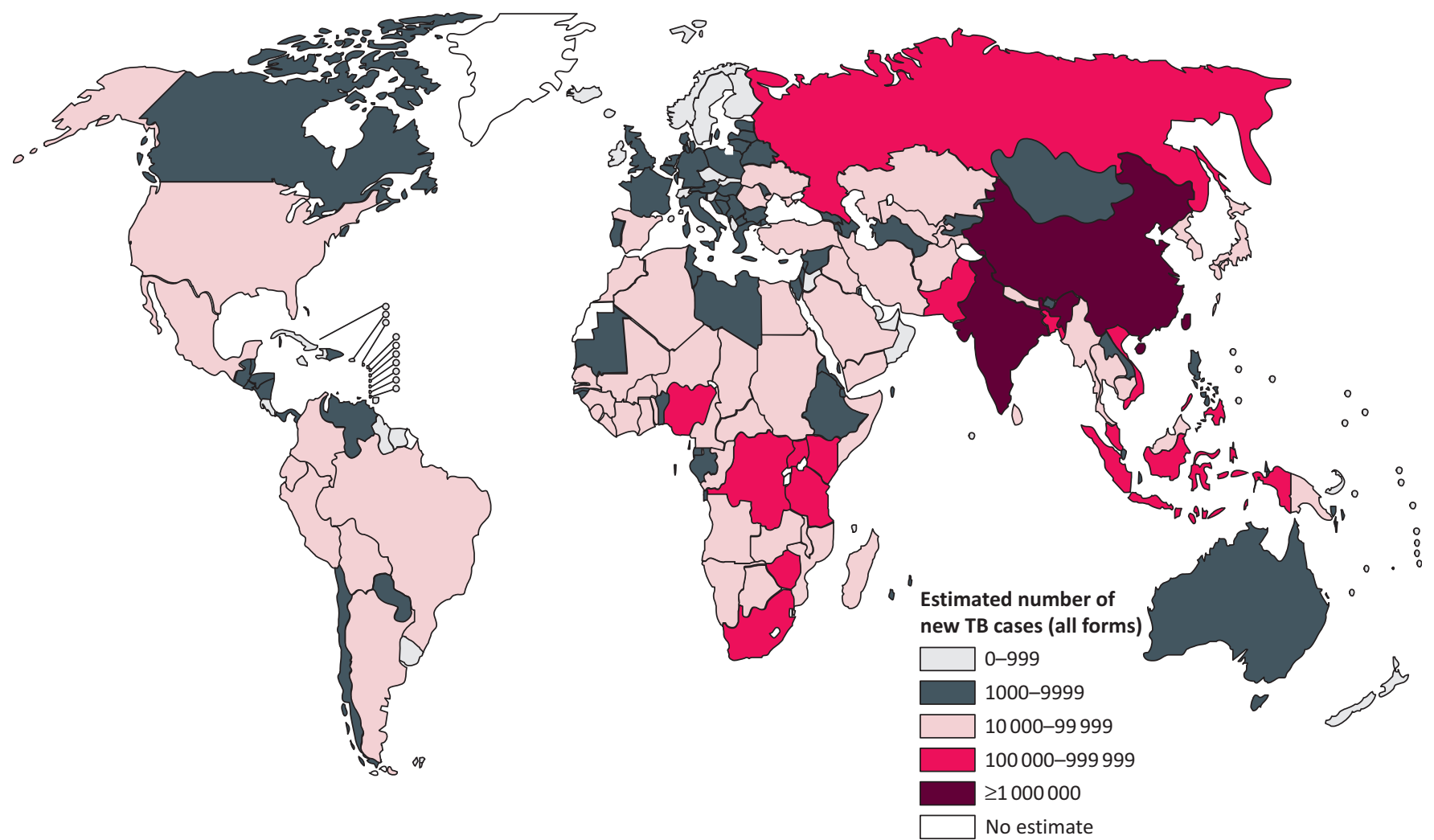

Fig 1. The number of new cases of tuberculosis worldwide in 2007. Reproduced with permission from the World Health Organization.

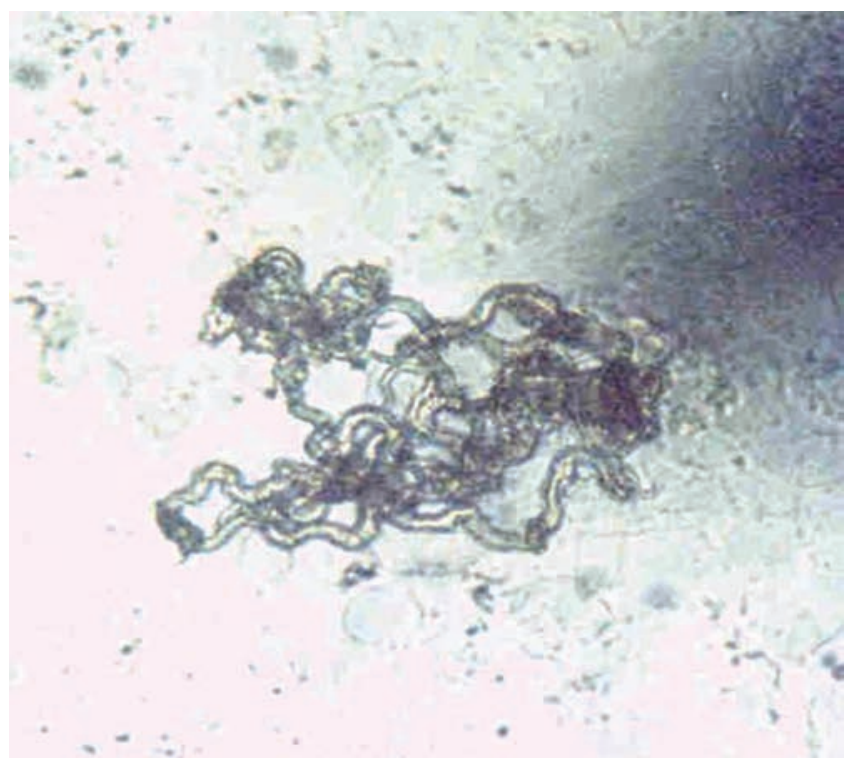

Fig 2. The typical appearance of Mycobacterium tuberculosis as see down the inverted light microscope during the MODS assay.

negative. MODS is relatively easy to learn and potential use of mobile phone and other technology may allow capturing of images with remote analysis in a low cost, highly effective way suitable for smaller diagnostic facilities.

\section{New anti-tuberculous drugs}

The first question is do we need any new anti-TB drugs since the majority of people who currently die with TB actually have fully sensitive disease which has led some to advocate focusing on finding and diagnosing disease. However, there are many issues around the current duration of therapy required with so-called short course treatment taking six months as a minimum. Treatment is often complicated by drug interactions, particularly in patients on highly active antiretroviral therapy (HAART) and by a range of side effects the most common of which is hepatotoxicity. However, the critical issue is the rise of drug resistance. Multi-drug resistant TB (MDR-TB) is defined as resistance to isoniazid and rifampicin and the $\mathrm{WHO}$ estimates that there are over 500,000 cases of MDR-TB worldwide which some believe is a conservative figure. In addition, there are many patients who have resistance to multiple anti-TB drugs. This first hit the medical headlines in 2006 when extensively-drug resistant TB or XDR-TB was defined as MDR-TB plus resistance to a quinolone and to at least one injectable anti-TB therapy was identified in South Africa. ${ }^{6}$ XDR-TB is on the rise (Fig 3) and this is despite the difficulty that many countries have in diagnosing resistance to first line, never mind second line, antimycobacterial drugs. The rise of drug-resistant TB has implications not only for the patient with active disease but also to the treatment of latent infection which may now often no longer be 


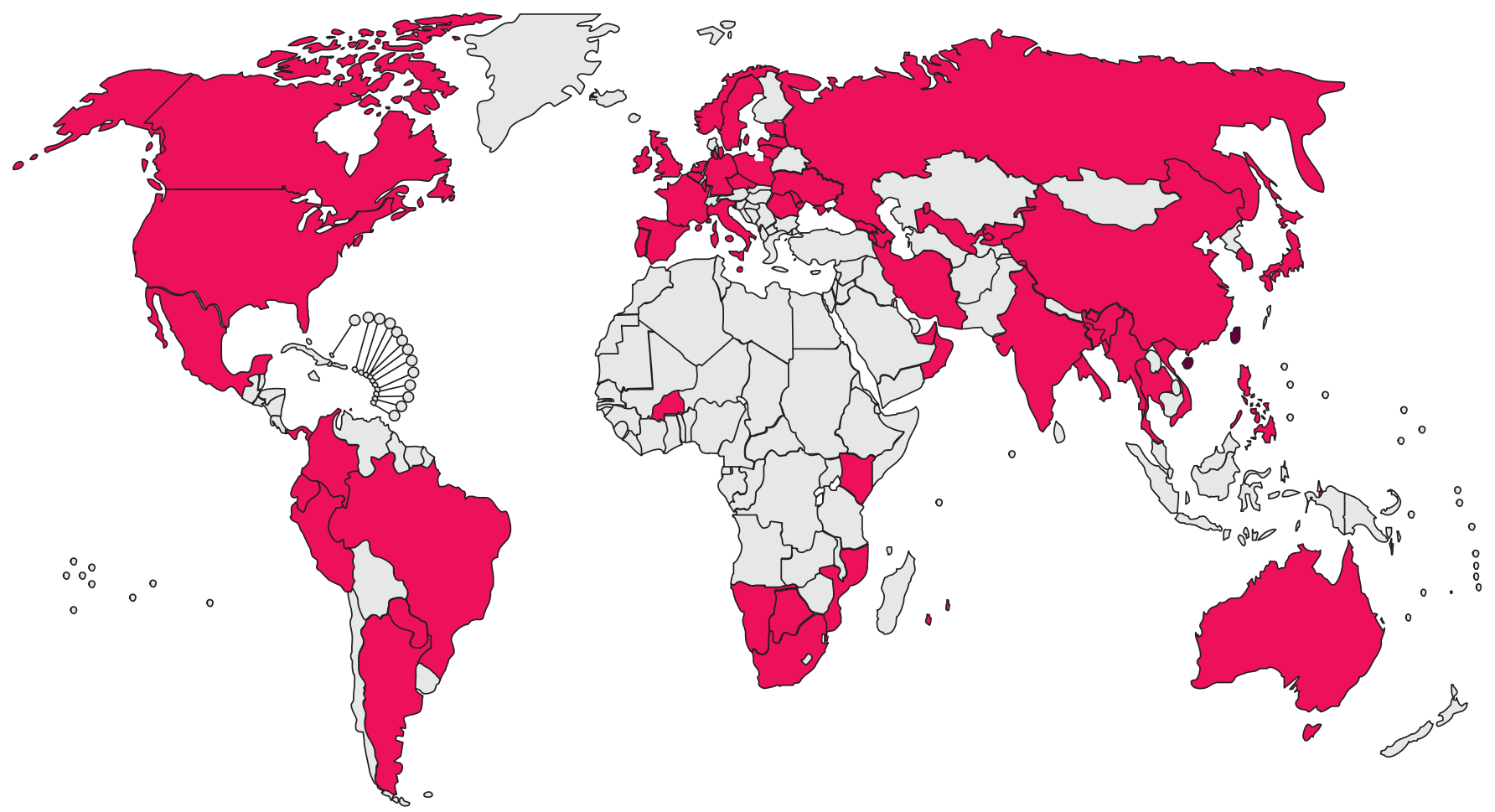

$\begin{array}{lllllll}\text { Argentina } & \text { Burkina Faso } & \text { Georgia } & \text { Kenya } & \text { Nepal } & \text { Qatar } & \text { Swaziland } \\ \text { Armenia } & \text { Canada } & \text { Germany } & \text { Latvia } & \text { Netherlands } & \text { Republic of Korea } & \text { Sweden } \\ \text { Australia } & \text { China } & \text { India } & \text { Lesotho } & \text { Norway } & \text { Republic of Moldova } & \text { Thailand } \\ \text { Azerbaijan } & \text { Colambia } & \text { Iran } & \text { Lithunia } & \text { Oman } & \text { Romania } & \text { Ukraine } \\ \text { Bangladesh } & \text { Czech Republic } & \text { Ireland } & \text { Mexico } & \text { Peru } & \text { Russian Federation } & \text { United Arabh Emirates } \\ \text { Belgium } & \text { Ecudor } & \text { Israel } & \text { Mozambique } & \text { Philippines } & \text { Slovenia } & \text { United Kingdom } \\ \text { Botswana } & \text { Estonia } & \text { Italy } & \text { Myanmar } & \text { Poland } & \text { South Africa } & \text { United States of America } \\ \text { Brazil } & \text { France } & \text { Japan } & \text { Namibia } & \text { Portugal } & \text { Spain } & \text { Uzbekistan } \\ & & & & & & \text { Veitnam }\end{array}$

Fig 3. The number of countries reporting XDR-TB by late 2009. This does not take account of restricted laboratory diagnostic facilities in many parts of the world. Reproduced with permission from the World Health Organization.

sensitive to the single drug therapy given to this quiescent form of infection. Treatment of latent tuberculosis is a separate and complex area where a central problem in resource poor circumstances is ensuring that active disease is not being inadvertently treated with a single drug.

What would the ideal new anti-tuberculous drug look like? Ideally it should kill MTb via a new target, be more potent than present drugs and able to reduce duration of therapy. Studies are ongoing to see if anti-TB therapy can be reduced to four months but if a major impact is to be made on disease burden, therapy needs to be under a month long. New drugs should be active against latent and multiplying organisms including those with drug resistance. Ideally a new drug should not be toxic and would have minimal drug interactions, particularly with HAART. Finally, the mythical new drug should be given orally with infrequent dosing.

The reality is that the new drug pipeline for TB is very small. ${ }^{7}$ Many recent efforts have concentrated on the use of old drugs in new ways. Moxifloxacin has recently been shown to reduce the time to culture negativity compared to ethambutol in a quadruple drug regiment. ${ }^{8}$ Close inspection has also shown that rifampicin is used at a relatively low dose and that higher doses (900-1200 mg) may lead to shorter course chemotherapy. The longer acting rifamycin, rifapentene has less drug interactions but is also a poorer drug than the parent rifampicin. Other antibiotics for traditional bacterial infections are being investigated for anti-TB activity particularly in patients who have drug resistant organisms. For example, linezolid has good in vitro activity against MTb although resistance may develop and prolonged in vivo use is associated with side effects. ${ }^{9}$

The diarylquinolines are the most developed new drug category discovered by high throughput screening of substances which inhibited the growth of $M$. Smegmatis..$^{10}$ The drug target is novel being the proton pump $\mathrm{F}_{0}$ subunit of mycobacterial ATPase. The drug (TMC-207) has a long half life and appears well tolerated in man where it is being used in patients with drug-resistant disease. The second class of new drugs are the nitroimidazoles, of which the best characterised example goes under the name PA- 824 which is a pro-drug activated by mycobacterial enzymes. The mechanism of action is not fully 
understood but it does not appear to cross resist with other antiTB drugs. Interestingly, the drug appears to work well in hypoxia against non-dividing organisms which may be relevant in continuation phase therapy with organisms in necrotic tissue. However, it does not appear to shorten the duration of therapy required to cure TB. Among the other drugs that are waiting in the wings are diamines, pyrroles and benzothiazinones which are all at early stages of development but the list of new drugs is limited.

It is now time to think about new approaches to TB treatment. Since the human immune system copes so well with MTb infection most of the time and keeps the organism contained within the host, it may be that it will be possible to harness the immune response to overcome or limit active infection. This may sound farfetched but changes in technology allow therapies that seem initially expensive to move to the clinic surprisingly quickly particularly if they are to deal with otherwise life threatening drugresistant disease. In addition, it seems that we have unknowingly been using immunotherapy for over 60 years. We showed that para-aminosalycilic acid (PAS), a second lined anti-TB drug, actually acts to block prostaglandin-dependent pathways which drive tissue destruction in TB before it has any effect on inhibiting mycobacterial growth. ${ }^{11}$ Potentially new therapies might target the enzymes that cause tissue damage in patients. ${ }^{12}$

\section{Biomarkers and TB}

A biomarker can be defined as a measured characteristic(s) which indicates normal or pathological biological responses or a response to a treatment or other intervention. Biomarkers come in many different forms and may not be of functional importance. Biomarkers are potentially useful in TB diagnosis, prognosis including the prediction of relapse, the assessment of drug resistance and other treatment responses as well as the development of adverse reactions such as the immune reconstitution syndrome in HIV TB. Finally, biomarkers may be key in monitoring vaccine responses where currently massive trials are required to reach clinical end points such as disease prevention.

Biomarkers have always been part of clinical practice but changes in symptoms such as fever, night sweats and cough are not specific. Chest X-rays, skin tests and quantitative sputum analysis have all been proposed as biomarkers and found wanting in terms of sensitivity and specificity. Interferon gamma release assays do not distinguish active and latent disease or reliably change following treatment of infection. A number of single host immunomodulatory proteins have been suggested as biomarkers ranging from C-reactive protein through tumour necrosis factor and interferon-inducible protein 10 as well as more complex biomarkers such as whole blood MTb killing. No single biomarker has proved robust and using multiple biomarkers on the basis of hypothesis driven algorithms have been disappointing. Biomarkers based on MTb antigens in sputum or urine have also not been reliable. A more inclusive approach is required which ideally does not make assumptions about disease processes. To this end, proteomics, genomics and metabolomics are novel technologies which hold out promise for the future. ${ }^{13}$ There are many practical issues associated with such - omic markers relating to the large number of variables compared to the number of samples, the way samples are collected and the effect of tissue specificity and leukocyte migration. In addition, there are mathematical issues around data over-fitting and other aspects of number crunching. Thus, findings at present require verification by a second approach. Proteomic technology is currently being used to distinguish active from latent TB. In the medium term, proteomic studies lead to identification of specific proteins that characterise a disease state with the aim of transferring detection away from mass spectrometry to dipstick technology. Experience with the national lottery tells most people that you do not need too many proteins to have a highly specific read out.

\section{Final thoughts}

TB remains a major problem worldwide and patient numbers are continuing to rise in the UK. Rapid diagnosis is the pressing need with drug sensitivity data. Although molecular tests may hold promise for the future, MODS is an attractive easily implemented technology. In the face of the exponential rise of drugresistant $\mathrm{TB}$, there are some, but not many, interesting new drugs on the horizon as well as a few new roles for existing antibiotics. The question is whether it will be possible to exploit immunomodulation as may already have been done. The biomarker field is exciting and may facilitate diagnostic treatment and vaccine studies but this is very much work in progress. TB remains a great conundrum and a fascinating clinical disease with many areas requiring more research. In the 21st century, more clinicians need to respond to the call and find the best ways to defeat an enduring pathogen.

\section{Acknowledgements}

The author's work is supported by diverse grant funding, particularly from the Medical Research Council (UK) and The Wellcome Trust. The author acknowledges support from the National Institute for Health Research (NIHR) Biomedical Research Centre Funding Scheme at Imperial College London.

\section{References}

1 Boehme CC, Nabeta P, Hillemann D et al. Rapid molecular detection of tuberculosis and rifampin resistance. $N$ Engl J Med 2010;363:1005-15.

2 Wallis RS, Pai M, Menzies D et al. Biomarkers and diagnostics for tuberculosis: progress, needs, and translation into practice. Lancet 2010;375:1920-37.

3 Caviedes L, Lee TS, Gilman RH et al. Rapid, efficient detection and drug susceptibility testing of Mycobacterium tuberculosis in sputum by microscopic observation of broth cultures. The Tuberculosis Working Group in Peru. J Clin Microbiol 2000;38:1203-8.

4 Minion J, Leung E, Menzies D, Pai M. Microscopic-observation drug susceptibility and thin layer agar assays for the detection of drug resistant tuberculosis: a systematic review and meta-analysis. Lancet Infect Dis 2010;10:688-98. 
5 Moore DA, Evans CA, Gilman RH et al. Microscopic-observation drug-susceptibility assay for the diagnosis of TB. N Engl J Med 2006;355:1539-50.

6 Gandhi NR, Moll A, Sturm AW et al. Extensively drug-resistant tuberculosis as a cause of death in patients co-infected with tuberculosis and HIV in a rural area of South Africa. Lancet 2006;368:1575-80.

7 van den Boogaard J, Kibiki GS, Kisanga ER, Boeree MJ, Aarnoutse RE. New drugs against tuberculosis: problems, progress, and evaluation of agents in clinical development. Antimicrob Agents Chemother 2009;53:849-62.

8 Conde MB, Efron A, Loredo C et al. Moxifloxacin versus ethambutol in the initial treatment of tuberculosis: a double-blind, randomised, controlled phase II trial. Lancet 2009;373:1183-9.

9 Condos R, Hadgiangelis N, Leibert E et al. Case series report of a linezolid-containing regimen for extensively drug-resistant tuberculosis. Chest 2008;134:187-92.

10 Andries K, Verhasselt P, Guillemont J et al. A diarylquinoline drug active on the ATP synthase of Mycobacterium tuberculosis. Science 2005;307:223-7.
11 Rand L, Green JA, Saraiva L, Friedland JS, Elkington PT. Matrix metalloproteinase- 1 is regulated in tuberculosis by a p38 MAPK-dependent, p-aminosalicylic acid-sensitive signaling cascade. J Immunol 2009;182:5865-72.

12 Elkington PT, D’Armiento JM, Friedland JS. Tuberculosis immunopathology: the neglected role of extracellular matrix destruction. Sci Transl Med 2011;3:1-4.

13 Agranoff D, Fernandez-Reyes D, Papadopoulos MC et al. Identification of diagnostic markers for tuberculosis by proteomic fingerprinting of serum. Lancet 2006;368:1012-21.

Address for correspondence: Professor JS Friedland, Department of Infectious Diseases, Hammersmith Campus, Imperial College London, Du Cane Road, London, W12 0NN. Email: j.friedland@imperial.ac.uk

\section{Local adult neurology services for the next decade}

\section{Report of a working party}

Neurological disorders are very common, accounting for about one in ten general practitioner consultations, around $10 \%$ of emergency medical admissions (excluding stroke) and disability for one in 50 of the UK population.

Patients require access to different parts of the neurological care pathway at different stages of their illness (acute admission, outpatient care and long-term care). However, these are currently poorly planned and organised. Good management requires better integrated primary, secondary and tertiary resources to achieve a neurology network that is easily accessible, provides local care where appropriate and, when necessary, involves the regional neurosciences centre.

Highlighting the need for acute neurology services in district general hospitals and the community, this report emphasises the value of multidisciplinary interaction with nurses and GPs, and urges a better structured and integrated approach. Other improvements proposed include a rise in the number of district general hospital neurologists, and dedicated nursing staff and neurology wards. The report also stresses the need for neurologists to take an active role in the planning and commissioning of services. :

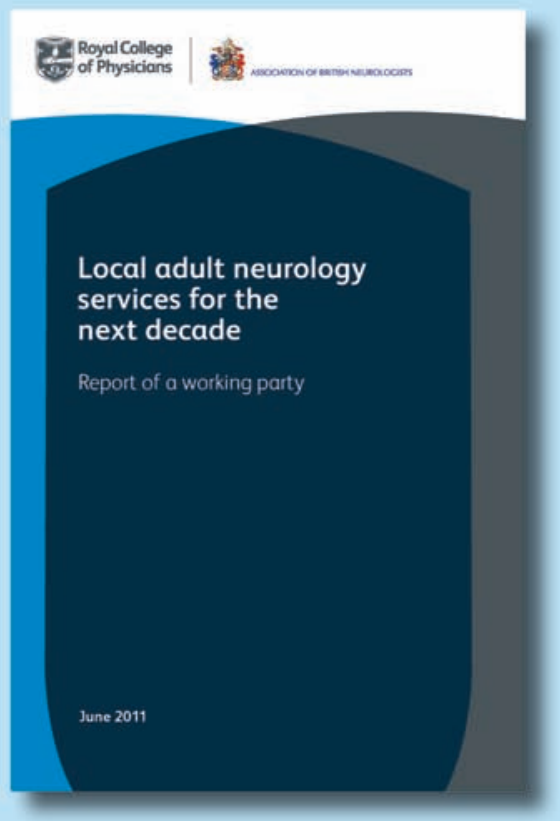

Published: May 2011 ISBN: 9781860164446

Price: $£ 5.00$ UK, $£ 7.00$ overseas (inc post and packing) 